\title{
Topological surface plasmons in superlattices with changing sign of the average permittivity
}

\author{
Hanying Deng ${ }^{1}$, Xianfeng Chen ${ }^{1}$, Nicolae C. Panoiu ${ }^{2}$, and Fangwei Ye $^{1 *}$ \\ ${ }^{1}$ Department of Physics and Astronomy, Shanghai Jiao Tong University, 800 Dongchuan Road, Shanghai 200240, China \\ ${ }^{2}$ Department of Electronic and Electrical Engineering, University College London, Torrington Place, London WC1E7JE, United Kingdom \\ *Corresponding author: fangweiye@sjtu.edu.cn
}

Compiled August 20, 2016

\begin{abstract}
We address the topological properties of onedimensional plasmonic superlattices composed of alternating metallic and dielectric layers. We reveal that the Zak phase of such plasmonic lattices is determined by the sign of the spatial average of their permittivity, $\bar{\varepsilon}$, and as such the topology and their associated interfacial (edge) states are extremely robust against structural disorder. Our study shows that the topologically protected interfacial modes occurring at the interface between two plasmonic lattices with opposite sign of $\bar{\varepsilon}$ can be viewed as the generalization of the conventional surface plasmon polaritons existing at metallic-dielectric interfaces.

(C) 2016 Optical Society of America
\end{abstract}

OCIS codes: (350.4238) Nanophotonics and photonic crystals; (310.6626) Subwavelength structures, nanostructures; (240.6680) Surface plasmons; (240.6648) Surface dynamics.

\section{http://dx.doi.org/10.1364/ao.XX.XXXXXX}

The nontrivial topological properties of matter have been attracting increasing interest in condensed matter physics, as the theory of band topology explains a series of striking phenomena like quantum Hall effect [1-3] and topological insulators [4-8]. Starting from the duality between their mathematical description, similar concepts and ideas have been introduced into the realm of optics and nontrivial topological effects have been demonstrated across a variety of optical systems [9]. Perhaps the simplest optically topological structure is analogous to the celebrated Su-Schrieffer-Heeger (SSH) model for polyacetylene [10], in which a chain of sites with alternating sign of the coupling constant exhibits two topologically distinct phases and topologically protected interfacial modes exist at the interface between two topologically distinct chains. Realization of the $\mathrm{SSH}$ model in photonic systems includes dimerized dielectric waveguides [11] and dielectric nanoparticles [12], as well as metallic nanodisks [13]. Furthermore, edge states between coupled plasmonic waveguides described by the SSH model have been investigated in graphene [14] and plasmonic waveguide ar- rays [15]. These structures are optically discrete thus they closely mimic the original SSH model. On the other hand, topological properties and associated edge states of one-dimensional (1D) photonic structures have also been investigated in continuous periodic systems, i.e., beyond the tight-binding approximation and other discrete models $[16,17]$.

All the topological properties of 1D structures mentioned above can be characterized by a single physical quantity, the so-called Zak phase [18]. This is a special kind of Berry phase, associated to $1 \mathrm{D}$ bulk bands. The characterization of the Zak phase of matter is of fundamental importance to the understanding of topology related physical properties of condensed matter systems. Optics is emerging as an alternative platform to study such topology induced phenomena, as in many cases it provides more suitable theoretical and experimental tools to explore them. In particular, specific ways to measure the Zak phase in optical systems have been proposed theoretically [19] and implemented experimentally by employing optical waveguide systems [20-23].

In this Letter, we focus on topological properties of 1D plasmonic superlattices composed of alternating metallic and dielectric layers. We reveal that the Zak phase of such superlattices is determined by the sign of the spatial average of the permittivity of the lattice, $\bar{\varepsilon}$, such that lattices with $\bar{\varepsilon}>0$ are topologically distinct from those with $\bar{\varepsilon}<0$. Due to the fact that fully random structural perturbations of the superlattice that preserve the averaged values of the thickness of the constituent layers do not modify the value of $\bar{\varepsilon}$ and consequently its sign, the topology and the associated interfacial (edge) states of the plasmonic superlattices are found to be extremely robust against such perturbations. Our analysis reveals that the topologically protected modes at the interface between two superlattices with opposite sign of the average permittivity represent a conceptual generalization of the well-known surface plasmon polaritons (SPPs) formed at metal/dielectric interfaces.

Let us consider a 1D plasmonic superlattice composed of alternating layers of metallic and dielectric materials stacked along the $x$-axis, as depicted in Fig. 1(a). To be more specific, we assume that the metallic and dielectric layers are made of silver and silicon, respectively. At the wavelength of $\lambda=1550 \mathrm{~nm}$, the permittivity of dielectric (silicon) materials is $\varepsilon_{d}=12.25$ and the complex permittivity of the metal (silver) is $\varepsilon_{m}=-125.39+2.84 i$ [24]. As the imaginary part of the per- 
(a)
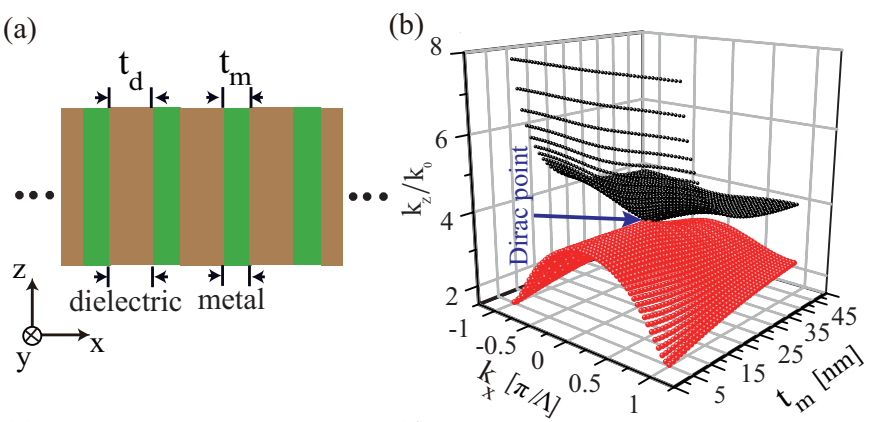

(c)

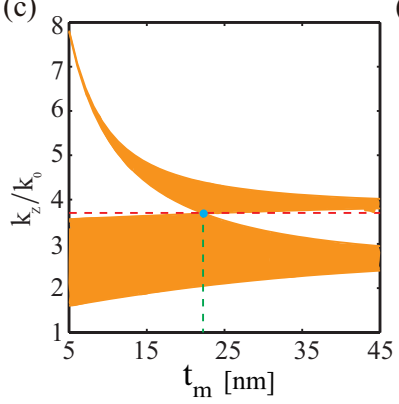

(d)

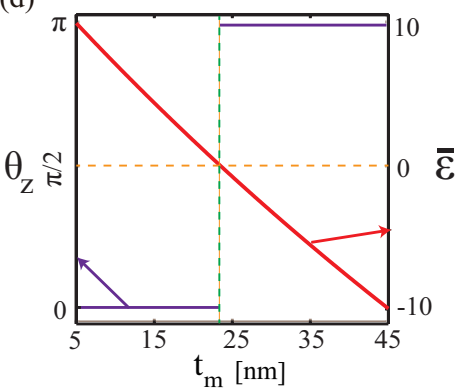

Fig. 1. (a) Schematic of a dielectric-metal layered lattice. (b) Dependence of photonic bandstructure $k_{z}\left(k_{x}\right)$ on the thickness of the metallic layer, $t_{m}$. A DP appears in the bandstructure at $t_{m}=23.45 \mathrm{~nm}$, for which $\bar{\varepsilon}=0$. (c) Lattice transmission bands (orange domains) vs. metallic layer thickness. (d) Average permittivity $\bar{\varepsilon}$ of the lattice vs. $t_{m}$. (e) Zak phase of the lattice vs. $t_{m}$. In these calculations, $\lambda=1550 \mathrm{~nm}, t_{d}=240 \mathrm{~nm}, \varepsilon_{m}=$ -125.39 and $\varepsilon_{d}=12.25$.

mittivity of the metal is very small as compared to its real part, the influence of the metal loss (heat dissipation) on the results are found to be negligible. Despite this, in the following analysis we have taken into account the small imaginary part of the permittivity of the metal, unless otherwise stated.

Considering the propagation along the z-axis of a TMpolarized optical beam (i.e., the only nonzero components of the eletromagnetic fields are $E_{x}, E_{z}$, and $H_{y}$ ), one can readily find the photonic bandstructure of the superlattice by solving the following transcendental equation:

$$
\begin{aligned}
\cos \left(k_{x} \Lambda\right)= & \cos \left(k_{d} t_{d}\right) \cos \left(k_{m} t_{m}\right) \\
& -\frac{1}{2}\left(\frac{\zeta_{d}}{\zeta_{m}}+\frac{\zeta_{m}}{\zeta_{d}}\right) \sin \left(k_{d} t_{d}\right) \sin \left(k_{m} t_{m}\right),
\end{aligned}
$$

where $k_{x}$ is the Bloch wavevector, $k_{z}$ is the propagation wavevector, $k_{j}=\sqrt{(\omega / c)^{2} \varepsilon_{j} \mu_{j}-k_{z}^{2}}, \zeta_{j}=k_{j} / \varepsilon_{j},(j=d, m), t_{d}$ and $t_{m}$ are the thickness of dielectric and metallic layers, respectively, $\Lambda=t_{d}+t_{m}$ is the period of the superlattice, and $c$ is the light speed in vacuum. By fixing the frequency $\omega$ in Eq. (1), the dependence $k_{z}=k_{z}\left(k_{x}\right)$ defines the photonic bandstructure (spatial dispersion relation) for that particular frequency [25]. The dependence of the bandstructure on the thickness of the metallic layer is shown in Fig. 1(b), for a fixed thickness of the dielectric layer, $t_{d}=240 \mathrm{~nm}$. Note that choosing a specific value for $t_{d}$ does not make our analysis less general. However, it should be mentioned that, in order for a two-band configuration to occur, as it is required in this study for reasons that will become apparent later, $t_{d}$ should be larger than a certain critical value (for example, for $t_{m}=25 \mathrm{~nm}$, the minimum $t_{d}$ required is $199 \mathrm{~nm}$ ).
A known property of the bandstructure of plasmonic superlattices is that, when the spatially averaged permittivity of the superlattice is zero, namely, when $\bar{\varepsilon}=\frac{\varepsilon_{d} t_{d}+\varepsilon_{m} t_{m}}{t_{d}+t_{m}}=0$, a diabolical point (DP) - the 1D counterpart of Dirac points - appears at the center of Brillouin zone, $k_{x}=0$ [25-27]. This property is illustrated in Figs. 1(b) and 1(c), as indeed for some specific value of $t_{m}$ (in the example shown in these plots, $t_{m}=23.45 \mathrm{~nm}$ ), the condition $\bar{\varepsilon}=0$ holds. Moreover, for this specific value of $t_{m}$, the upper band touches the lower one in such a way that a DP is formed at $\left(k_{x}, k_{z}\right)=\left(0, k_{z}^{\mathrm{DP}}\right)$, where $k_{z}^{\mathrm{DP}}=k_{0} \sqrt{\epsilon_{m} \epsilon_{d} /\left(\epsilon_{m}+\epsilon_{d}\right)}$. On the other hand, once the zero-average-permittivity condition is broken, namely when either $\bar{\varepsilon}>0$ or $\bar{\varepsilon}<0$, the DP vanishes and a gap opens. Upon understanding this mechanism of formation of a DP, an interesting question arises, i.e. in the context of light waves interaction with such superlattices what are the differences between a superlattice with $\bar{\varepsilon}>0$ and one with $\bar{\varepsilon}<0$ ?

To answer this question, we study the topological properties of these superlattices by calculating the Zak phase of their bulk bands, defined by the following formula [18]:

$$
\theta_{z}=\int_{-\pi / \Lambda}^{\pi / \Lambda}\left(i \int_{\text {unit cell }} \psi_{n, k_{x}}^{*} \frac{\partial \psi_{n, k_{x}}}{\partial k_{x}} d x\right) d k_{x}
$$

where $\psi_{n, k_{x}}$ is the periodic-in-cell part of the Bloch magnetic field eigenfunction of a state belonging to the $n$-th band at $k_{x}$, i.e., $H_{y, n, k_{x}}(x)=\psi_{n, k_{x}}(x) \exp \left(i k_{x} x\right)$. The function $\psi_{n, k_{x}}(x)$ can be obtained analytically using the transfer-matrix method [28]. The Zak phase depends on the choice of the origin, and here we choose this origin to be the center of the dielectric layer.

The outcome of the calculation of the Zak phase is shown in Fig. 1(d). As expected, the value of $\theta_{z}$ is binary valued, being equal to either 0 or $\pi$. Importantly, the Zak phase of the plasmonic superlattices is found to be solely dependent on the sign of the average permittivity, thus being independent on the particular geometry of the superlattice or the values of the permittivity of the layers. Specifically, it is equal to zero if $\bar{\varepsilon}>0$ and is equal to $\pi$ when $\bar{\varepsilon}<0$. In other words, the topology of plasmonic superlattices is uniquely defined by the sign of $\bar{\varepsilon}$ and structural transformations or geometrical fluctuations preserve the superlattice topology provided that $\bar{\varepsilon}$ does not change its sign. If, on the other hand, the plasmonic superlattice is structurally transformed in such a way that $\bar{\varepsilon}$ changes its sign after it passes through the zero point, as per Fig. $1(\mathrm{~d}), \theta_{z}$ varies accordingly leading to the modification of the topology of the superlattice. Note also that by changing the origin with respect to which the Zak phase is calculated the two constant values change. However, the same phase shift of $\pi$ occurs when $\bar{\varepsilon}$ changes its sign.

The dependence of the topology of plasmonic superlattices on the sign of $\bar{\varepsilon}$ can be explained by the general topological band theory. As mentioned, a DP appears in the bandstructure of lattices when $\bar{\varepsilon}=0$ and it vanishes (with a gap opening in the bandstructure) when $\bar{\varepsilon}$ becomes nonzero. Following the general topological band theory, which states that the topological phase of matter changes when a band gap closes at a DP and then reopens when the system is further modified $[4,5]$, we therefore expect that the topology of plasmonic superlattices is indeed characterized by the sign of $\bar{\varepsilon}$ and remains invariant as long as the sign of $\bar{\varepsilon}$ is preserved. Thus, a fundamental result of this analysis is that we can directly link in a simple way the topology of plasmonic superlattices to a single invariant parameter characterizing the plasmonic structure, namely, the 
(a)

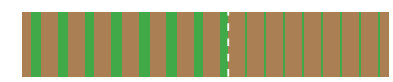

(b)
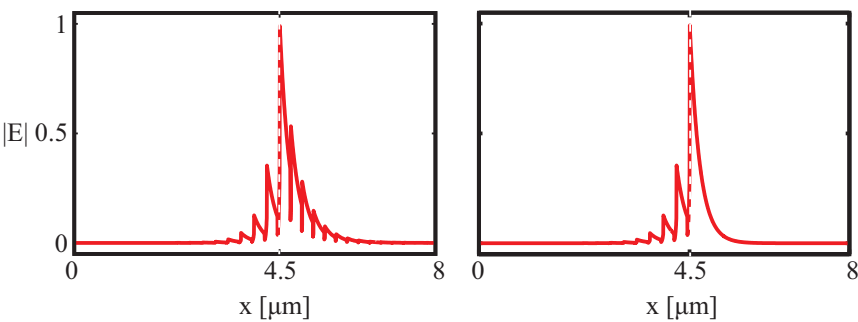

Fig. 2. (a) Profile of the electric field of the surface mode located at the interface between a plasmonic lattice with $t_{m}=45 \mathrm{~nm}(\bar{\varepsilon}=-9.48+0.45 i)$ and a plasmonic lattice with $t_{m}=10 \mathrm{~nm}(\bar{\varepsilon}=6.74+0.11 i)$. (b) The same as in (a) but with the second superlattice replaced by a uniform dielectric medium with $\varepsilon=12.25$. The white dashed lines indicate the position of the interface. The remaining parameters are $t_{d}=240 \mathrm{~nm}, \varepsilon_{m}=-125.39+2.84 i$, and $\varepsilon_{d}=12.25$.

sign of the average dielectric permittivity! This finding may have practical implications to the design and characterization of topologically functional devices implemented using plasmonic nanostructures. Equally important, these ideas can potentially be extended to other 1D superlattices characterized by spatially averaged rather than local physical quantities, such as zero- $\bar{n}$ superlattices [29-31]. Our findings also imply that, if for instance graphene sheets are incorporated in superlattices, electrically or optically tunable topological nanodevices can be achieved thanks to the tunability of graphene [27].

Consider now two adjacent, semi-infinite plasmonic superlattices and let us investigate the possibility that localized states exist at the interface between superlattices. The two superlattices are designed to have different sign of $\bar{\varepsilon}$ by, for example, using different thickness of the metallic layers, as illustrated in Fig. 2(a), or even setting $t_{m}=0$ for one superlattice, which means that the superlattice becomes a pure dielectric medium [see Fig. 2(b)]. Consistent with the bulk-edge correspondence principle, which states that localized zero-energy states exist at the interface between two insulators with distinct band topology, our mode analysis of such interfacial systems reveals that, if two connected superlattices differ in their sign of $\bar{\varepsilon}$, localized modes always appear at the interface separating the two superlattices. On the other hand, if the sign of $\bar{\varepsilon}$ for both superlattices is the same, their interface supports no localized modes. This is similar to the case of surface waves at the interface between two homogeneous and isotropic media, namely such localized modes, called SPPs, exist only if the permittivities of the two media have opposite sign. Indeed, the field profile of the surface modes in Fig. 2 resembles that of SPPs, the additional feature being the field oscillations in superlattices.

The above analysis is further corroborated by our direct beam propagation simulations. Specifically, we launched normally onto the interface a TM-polarized Gaussian beam of a narrow width, $w \approx 3 t_{d}$, and determined the electromagnetic field as it propagated into the superlattice. As expected, when the sign of $\bar{\varepsilon}$ in the two adjacent superlattices takes different values, a localized mode quickly forms at the interface while the extra energy of the input wave diffracts as radiative waves [see Figs. 3(a) and $3(b)]$. By contrast, when the sign of $\bar{\varepsilon}$ of the two adjacent (a)

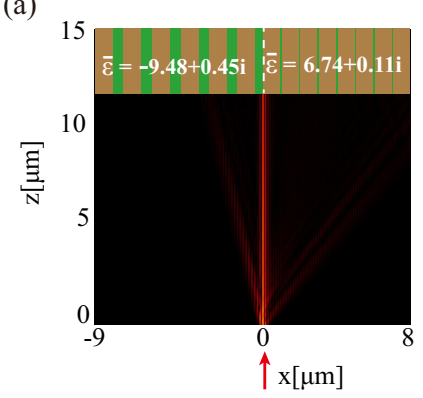

(b)

(c)

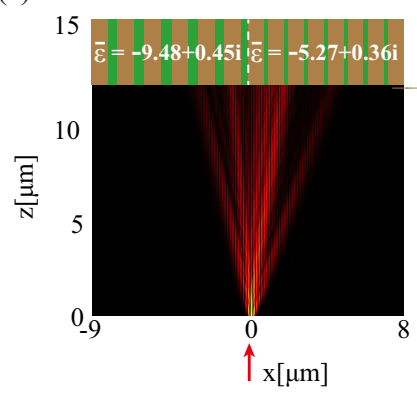

(d)
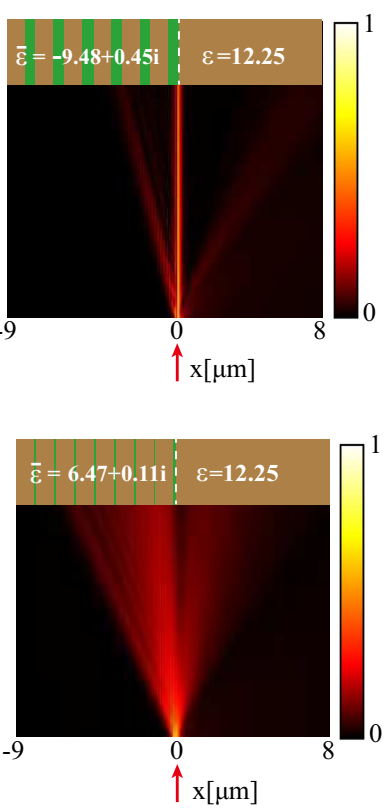

Fig. 3. Top (bottom) panels show the evolution of the normalized electric field amplitude when a TM-polarized Gaussian beam is injected normally at the interface between two plasmonic superlattices with opposite (the same) sign of $\bar{\varepsilon}$. In the right panels, one of the superlattices is replaced by a homogeneous medium. The average of the dielectric permittivity for each superlattice and medium is indicated on figure. The $E_{x}$ component of the input Gaussian beam is given by $E_{x}(x)=\exp \left[-x^{2} /\left(3 t_{d}\right)^{2}\right]$, whereas $t_{d}=240 \mathrm{~nm}$ is the thickness of the dielectric layers. Red arrows indicate the location of the incident beam.

superlattices is the same [negative in Fig. 3(c) and positive in Fig. 3(d)], the input optical beam strongly diffracts without any signature of the formation of a surface mode being observed.

As the topology of plasmonic superlattices is determined by the sign of the averaged permittivity, one expects that the topology and the associated interfacial states are extremely robust against structural disorder. This is expected because a fully random perturbation of the structure that preserves the averaged values of the thickness of the constituent layers does not modify the spatially averaged dielectric permittivity, nor its sign. To test this conjecture, we consider two adjacent superlattices, as shown in Figs. 2, but now introduce disorder into them by defining a random fluctuation of the thickness of the metallic components. Thus, the thickness of the $n$-th Ag layer in each plasmonic lattice is set to $t_{m}^{n}=t_{m 0}+\delta_{n}$, where $t_{m 0}$ is the average thickness and $\delta_{n}$ is a random value. We assume $\delta_{n}$ to be uniformly distributed in the interval of $[-\delta, \delta], 0<\delta<t_{m 0}$, hence the level of disorder can be characterized by the parameter, $\Delta \equiv \delta / t_{m 0}$. The spectra and field profile of the interface modes determined for increasing disorder strength, $\Delta$, are shown in Fig. 4.

It is known that incorporating disorder in a $1 \mathrm{D}$ lattice always leads to the formation of localized Anderson modes. Anderson modes strongly depend on the disorder strength, their degree of spatial localization increasing with the disorder strength, $\Delta$. The eigenvalue spectra of Anderson modes were statistically averaged over 50 randomly-perturbed superlattice configurations, the results being presented in Fig. 4(a). It shows that, as 
(a)

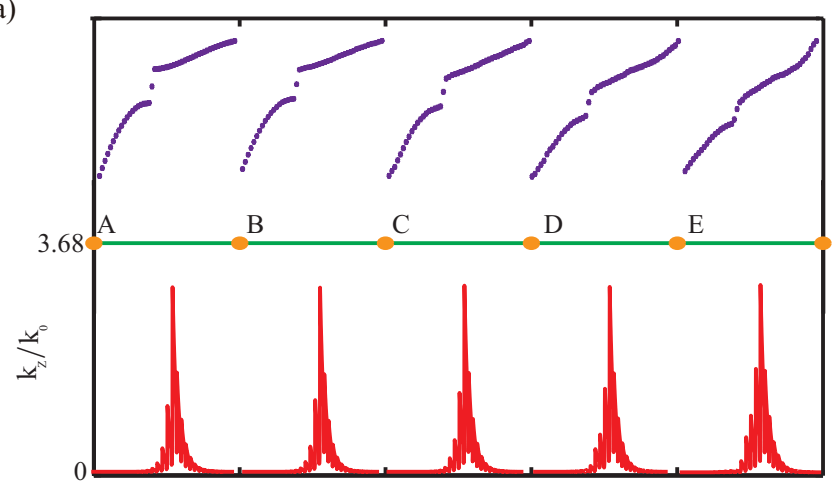

(b)

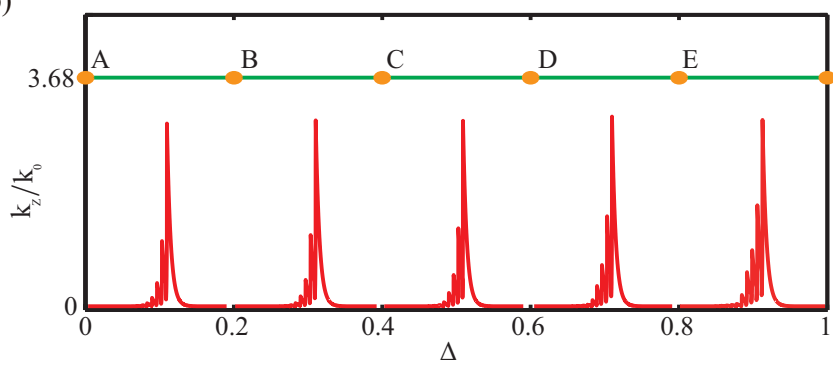

Fig. 4. Spectrum of Anderson modes (a) and dependence of eigenvalue (green line) and electric field amplitude (red curve) of the interfacial mode (b, c) on the disorder level, calculated for the interface system composed of two plasmonic lattices that, in the unperturbed limit, are the same as those shown in Fig. 2(a) and Fig. 2(b), respectively. The mode profiles and the spectrum of Anderson modes are calculated for five disorder levels: $0 \%$ (A), $20 \%$ (B), $40 \%$ (C), $60 \%$ (D), $80 \%(\mathrm{E})$. The dot inside the bandgap of Fig. 4(a) corresponds to the interface mode. All results are obtained by an ensemble-average over 50 disorder realizations.

expected, the Bragg gap of the unperturbed lattices narrows as $\Delta$ increases [32]. In sharp contrast, the wave profile of the interfacial mode remains almost unchanged, even when the disorder strength increases to $80 \%$ and above, as shown in Fig. 4(b) and Fig . 4(c). Furthermore, the eigenvalue $k_{z}$ (propagation constant) of the interfacial mode is unaffected by structural disorder. The interfacial mode is actually pinned down at the photonic DP of the unperturbed superlattice with $\bar{\varepsilon}=0$, namely, the eigenvalue of the interfacial mode is given by $k_{z}^{D P}=k_{0} \sqrt{\epsilon_{m} \epsilon_{d} /\left(\epsilon_{m}+\epsilon_{d}\right)}$. Note that this eigenvalue is equal to that of conventional SPPs formed at the interface between a semi-infinite metal and a semiinfinite dielectric medium [33]. Therefore, the topologically protected interface modes, localized at the interface between two superlattices characterized by different sign of $\bar{\varepsilon}$, represent a natural generalization of the conventional SPPs.Compared to conventional SPPs, however, such generalized SPP waves obviously have more degrees of freedom, which makes them an appealing alternative to be employed in nanophotonics applications. For example, by properly choosing the parameters of the two superlattices it could be possible to engineer and reduce the losses of topological SPPs below those of conventional SPPs.

In summary, we have studied the topological properties of plasmonic superlattices and revealed that their topology is determined by the sign of their spatially averaged dielectric permittivity. As such, their topology and the associated edge (interface) states are extremely robust against the structural random per- turbations. Such topologically protected localized states at the interface between two superlattices with opposite sign of the average permittivity represent a natural generalization of the well-known SPPs supported by metal/dielectric interfaces.

The work of H.D. and F.Y. was supported by Innovation Program of Shanghai Municipal Education Commission (Grant No. 13ZZ022) and the National Natural Science Foundation of China (Grant No. 61475101). F. Ye also acknowledges the support from Doctoral Program Foundation of Institutions of Higher Education of China(20110073120074). N.C.P. acknowledges support from European Research Council/ERC Grant Agreement No. ERC-2014-CoG-648328.

\section{REFERENCES}

1. D. Thouless, M. Kohmoto, M. Nightingale, and M. Den Nijs, Phys. Rev. Lett. 49, 405 (1982).

2. C. L. Kane and E. J. Mele, Phys. Rev. Lett. 95, 146802 (2005).

3. B. A. Bernevig, T. L. Hughes, and S.-C. Zhang, Science 314, 1757 (2006).

4. M. Z. Hasan and C. L. Kane, Rev. Mod. Phys. 82, 3045 (2010).

5. X.-L. Qi and S.-C. Zhang, Rev. Mod. Phys. 83, 1057 (2011).

6. L. H. Wu and X. Hu, Phys. Rev. Lett. 114, 223901 (2015).

7. H. Wang, L. Xu, H. Chen, and J. Jiang, Phys. Rev.B. 93, 235155 (2016).

8. L. Xu, H. Wang, Y. Xu, H. Chem, and J. Jiang, Opt. Express 24, 18059 (2016).

9. L. Lu, J. D. Joannopoulos, and M. Soljacic, Nat. Photonics 8, 821 (2014).

10. W. Su, J. R. Schrieffer, and A. J. Heeger, Phys. Rev. Lett. 42, 1698 (1979).

11. H. Schomerus, Opt. Lett. 38, 1912 (2013).

12. A. P. Slobozhanyuk, A. N. Poddubny, A. E. Miroshnichenko, P. A. Belov and Y. S. Kivshar, Phys. Rev. Lett. 114, 123901 (2015).

13. I. S. Sinev, I. S. Mukhin, A. P. Slobozhanyuk, A. N. Poddubny, A. E. Miroshnichenko, A. K. Samusev and Y. S. Kivshar, Nanoscale 7, 11904 (2015).

14. L. Ge, L. Wang, M. Xiao, W. Wen, and C. T. Chan and D Han, Opt. Express 23, 21585 (2015).

15. Q. Cheng, Y. Pan, Q. Wang, T. Li, and S. Zhu, Laser \& Photon. Rev. 9, 392 (2015).

16. N. Malkova, I. Hromada, X. Wang, G. Bryant and Z. Chen, Opt. Lett. 34, 1633 (2009).

17. M. Xiao, Z. Q. Zhang, and C. T. Chan, Phys. Rev. X 4, 021017 (2014).

18. J. Zak, Phys. Rev. Lett. 62, 2747 (1989).

19. S. Longhi, Opt. Lett. 38, 3716, 2013.

20. M. Atala, M. Aidelsburger, J. T. Barreiro, D. Abanin, T. Kitagawa, E. Demler, and I. Bloch, Nat. Phys. 9, 795 (2013).

21. A. Blanco-Redondo, I. Andonegui, M. J. Collins, G. Harari, Y. Lumer, M. C. Rechtsman, B. J. Eggleton, and M. Segev, Phys. Rev. Lett. 116, 163901 (2016)

22. W. S. Gao, M. Xiao, C. T. Chan, and W. T. Tam, Opt. Lett. 40, 5259 (2015).

23. Q. Wang, M. Xiao, H. Liu, S. Zhu, and C. T. Chan, Phys. Rev. B 93, 041415 (2016).

24. P. B. Johnson and R.-W. Christy, Phys. Rev. B 6, 4370 (1972).

25. S. H. Nam, A. J. Taylor, and A. Efimov, Opt. Express 18, 10120 (2010).

26. L. Sun, J. Gao, and X. Yang, Opt. Express 21, 021542 (2013).

27. H. Deng, F. Ye, B. A. Malomed, X. Chen, and N. C. Panoiu, Phys. Rev. B 91, 201402 (2015).

28. P. Yeh, A. Yariv, and C.-S. Hong, J. Opt. Soc. Am. 67, 423 (1977)

29. J. Li, L. Zhou, C. T. Chan, and P. Sheng, Phys. Rev. Lett. 90, 083901 (2003).

30. N. C. Panoiu, R. M. Osgood, S. Zhang, and S. R. J. Brueck, J. Opt. Soc. Am. B 23, 506 (2006).

31. S. Kocaman, R. Chatterjee, N. C. Panoiu, J. F. McMillan, M. B. Yu, R. M. Osgood, D. L. Kwong, and C. W. Wong, Phys. Rev. Lett. 102, 203905 (2009).

32. H. Deng, X. Chen, B. A. Malomed, N. C. Panoiu, and F. Ye, Sci. Rep. 5 (2015). 
33. S. Maier, Plasmonics: Fundamentals and Applications, Springer(2007). 


\section{REFERENCES}

1. D. Thouless, M. Kohmoto, M. Nightingale, and M. Den Nijs, "Quantized Hall conductance in a two-dimensional periodic potential," Physical Review Letters 49, 405 (1982).

2. C. L. Kane and E. J. Mele, " $Z 2$ topological order and the quantum spin Hall effect," Physical Review Letters 95, 146802 (2005).

3. B. A. Bernevig, T. L. Hughes, and S.-C. Zhang, "Quantum spin Hall effect and topological phase transition in $\mathrm{HgTe}$ quantum wells," Science 314, 1757 (2006).

4. M. Z. Hasan and C. L. Kane, "Colloquium: topological insulators," Reviews of Modern Physics 82, 3045 (2010).

5. X.-L. Qi and S.-C. Zhang, "Topological insulators and superconductors," Reviews of Modern Physics 83, 1057 (2011).

6. L. H. Wu and X. Hu, "Scheme for Achieving a Topological Photonic Crystal by Using Dielectric Material," Physical Review Letters 114, 223901 (2015).

7. H. Wang, L. Xu, H.. Chen, and J.. Jiang, "Three-dimensional photonic Dirac points stabilized by point group symmetry," Physical Review B 93, 235155 (2016).

8. L. Xu, H. Wang, Y. Xu, H. Chem, and J. Jiang, "Accidental degeneracy in photonic bands and topological phase transitions in twodimensional core-shell dielectric photonic crystals," Optics Express 24, 18059 (2016).

9. L. Lu, J. D. Joannopoulos, and M. Soljacic, "Topological photonics," Nature Photonics 8, 821 (2014).

10. W. Su, J. R. Schrieffer, and A. J. Heeger, "Solitons in polyacetylene," Physical Review Letters 42, 1698 (1979).

11. H. Schomerus, "Topologically protected midgap states in complex photonic lattices," Optics Letters 38, 1912 (2013).

12. A. P. Slobozhanyuk, A. N. Poddubny, A. E. Miroshnichenko, P. A. Belov and Y. S. Kivshar, "Subwavelength topological edge States in optically resonant dielectric structures," Physical Review Letters 114, 123901 (2015).

13. I. S. Sinev, I. S. Mukhin, A. P. Slobozhanyuk, A. N. Poddubny, A. E. Miroshnichenko, A. K. Samusev and Y. S. Kivshar, "Mapping plasmonic topological states at the nanoscale," Nanoscale 7, 11904 (2015).

14. L. Ge, L. Wang, M. Xiao, W. Wen, and C. T. Chan and D Han, "Topological edge modes in multilayer graphene systems," Optics Express 23, 21585 (2015)

15. Q. Cheng, Y. Pan, Q. Wang, T. Li, and S. Zhu, "Topologically protected interface mode in plasmonic waveguide arrays," Laser\& Photonics Reviews 9, 392 (2015).

16. N. Malkova, I. Hromada, X. Wang, G. Bryant and Z. Chen, "Observation of optical Shockley-like surface states in photonic superlattices,"' Optics Letters 34, 1633 (2009).

17. M. Xiao, Z. Q. Zhang, and C. T. Chan, "Surface impedance and bulk band geometric phases in one-dimensional systems," Physical Review X 4, 021017 (2014).

18. J. Zak, "Berry's phase for energy bands in solids," Physical Review Letters 62, 2747 (1989).

19. S. Longhi, "Zak phase of photons in optical waveguide lattices,", Optics Letters. 38, 3716, 2013.

20. M. Atala, M. Aidelsburger, J. T. Barreiro, D. Abanin, T. Kitagawa, E. Demler, and I. Bloch, "Direct measurement of the Zak phase in topological Bloch bands,", Nature Physics 9, 795 (2013).

21. A. Blanco-Redondo, I. Andonegui, M. J. Collins, G. Harari, Y. Lumer, M. C. Rechtsman, B. J. Eggleton, and M. Segev, "Topological Optical Waveguiding in Silicon and the Transition between Topological and Trivial Defect States,"” Physical Review Letters 116, 163901 (2016)

22. W. S. Gao, M. Xiao, C. T. Chan, and W. T. Tam, "Determination of Zak phase by reflection phase in 1D photonic crystals," Optics Letters 40, 5259 (2015).

23. Q. Wang, M. Xiao, H. Liu, S. Zhu, and C. T. Chan, "Measurement of the Zak phase of photonic bands through the interface states of a metasurface/photonic crystal," Physical Review B 93, 041415 (2016).

24. P. B. Johnson and R.-W. Christy, "Optical constants of the noble metals," Physical Review B 6, 4370 (1972).

25. S. H. Nam, A. J. Taylor, and A. Efimov, "Diabolical point and conical-like diffraction in periodic plasmonic nanostructures," Optics Express 18, 10120 (2010).

26. L. Sun, J. Gao, and X. Yang, "Giant optical nonlocality near the Dirac point in metal-dielectric multilayer metamaterials," Optics Express 21, 021542 (2013)

27. H. Deng, F. Ye, B. A. Malomed, X. Chen, and N. C. Panoiu, "Optically and electrically tunable Dirac points and Zitterbewegung in graphenebased photonic superlattices," Physical Review B 91, 201402 (2015).

28. P. Yeh, A. Yariv, and C.-S. Hong, "Electromagnetic propagation in periodic stratified media. I. General theory," Journal of the Optical Society of America 67, 423 (1977).

29. J. Li, L. Zhou, C. T. Chan, and P. Sheng, "Photonic band gap from a stack of positive and negative index materials," Phys. Rev. Lett. 90, 083901 (2003).

30. N. C. Panoiu, R. M. Osgood, S. Zhang, and S. R. J. Brueck, "Zero- $\bar{n}$ bandgap in photonic crystal superlattices," J. Opt. Soc. Am. B 23, 506 (2006).

31. S. Kocaman, R. Chatterjee, N. C. Panoiu, J. F. McMillan, M. B. Yu, R. M. Osgood, D. L. Kwong, and C. W. Wong, "Observation of Zeroth-Order Band Gaps in Negative-Refraction Photonic Crystal Superlattices at Near-Infrared Frequencies," Phys. Rev. Lett. 102, 203905 (2009).

32. H. Deng, X. Chen, B. A. Malomed, N. C. Panoiu, and F. Ye, "Transverse Anderson localization of light near Dirac points of photonic nanostructures," Scientific Reports 5 (2015).

33. S. Maier, Plasmonics: Fundamentals and Applications, Springer(2007). 